\title{
Epidemiology of NDM-1 and its Variants in Multidrug Resistant Gram- Negative Bacilli Isolated from Infection in Cancer Patients
}

Hemant J Vira* and Vivek G Bhat

Department of Microbiology, ACTREC-Tata Memorial Centre, Mumbai, Maharashtra, India

\begin{abstract}
Infectious diseases are a major cause of morbidity and mortality throughout the world. Cancer patients are immunocompromised due to the disease itself and also due to multiple factors such as chemotherapy, radiotherapy, impairment of normal leukocyte function, and use of corticosteroids. This leads to development of resistant pathogens in them of which the New Delhi metallo- $\beta$-lactamase-1 (NDM-1) is mostly resistant to the drug of last resort the class of carbapenems. The increase in extended spectrum carbapenem resistance class of antibiotic has led to a worldwide need for a development of a more efficient and novel class of drug active against NDM-1 producing gram-negative bacteria. The epidemiological study of NDM-1 gene has revealed a startling fact on the widespread prevalence of extended spectrum carbapenem resistant gram-negative bacteria in cancer patients both nationally and internationally. Pharmacovigilance and formulations of different class of antibiotics are needed to tackle the problem of this widespread resistance. The various drug delivery methods and drug designing platforms are needed for the development of a novel class of drug needed to efficiently eradicate these multidrug resistant gram-negative pathogens. In the present review, we have described the epidemiological prevalence of NDM-1 and its variants producing gram negative bacilli in cancer patients both nationally and internationally, the problem of ineffectiveness of carbapenem class of antibiotics against such pathogens, and also the future line of developments needed in the field of pharmacovigilance and pharmacotherapeutics to develop a highly efficient, target specific, and cost-effective class of antibiotics.
\end{abstract}

Keywords: NDM-1; Gram negative; Cancer; Carbapenem

\section{Introduction}

Infections are a major cause of morbidity and mortality in cancer patients. The cancer patient is immunocompromised because of the nature of the disease itself and also due to interventions in the form of chemotherapy. In addition, there are usually other associated risk factors for acquiring infection such as long term catheterization, mucositis due to cytotoxic agents, neutropenia, and stem cell transplantation. This increased risk of bacterial infections is further compounded by the rising trends of antibiotic resistance in commonly implicated organisms all over the world. This is particularly true in the case of members of Enterobacteriaceae group like Escherichia coli and Klebsiella pneumoniae and the non-fermenter group of organisms such as Pseudomonas aeruginosa and Acinetobacter spp. in the Indian setting. There is already widespread resistance to the cephalosporins as shown by ESBL (extended spectrum $\beta$-lactamase) and Amp C producers among the Enterobacteriaceae. Rampant use of antibiotics has unfortunately led to increasing resistance to the carbapenems as well, and this is generally due to carbapenemase production by the organisms [1]. Prevalence of Metallo-beta-lactamase (MBL) producing organisms including New Delhi MBL-1 (NDM-1) is also on the rise in India. NDM- 1 (New Delhi metallo- $\beta$-lactamase- 1$)$ is the most recently discovered transferable molecular class B (zinc metallo-) $\beta$-lactamase. It is a monomeric metalloenzyme with a molecular mass of $28 \mathrm{kDa}$ [2]. It was described in Klebsiella pneumoniae and Escherichia coli isolated in Sweden in 2008 from an Indian patient transferred from a New Delhi hospital. Similar to other class B $\beta$-lactamases like IMP and VIM enzymes, it can hydrolyze all penicillins, cephalosporins, and carbapenems except the monobactam aztreonam [2]. Although NDM1 does not hydrolyze aztreonam, over $80 \%$ of bacteria with the enzyme are resistant, reflecting co-production of ESBLs (mostly CTX-M types) and plasmid-mediated Amp C cephalosporins [3].

The current emergence of carbapenemase-producing gramnegatives is of great concern because it is often associated to the occurrence of multidrug-resistant isolates for which very few (if any) antibiotic options remain available.

\section{International Status}

After the first report of NDM-1, it was found in many countries like UK, India, Pakistan and Bangladesh in 2008-2009 and reported in mid-August 2010 [4]. Since then NDM-1 positive bacteria have been reported worldwide, except in Central and South America $[5,6]$. Recently in an eReview, the data which was published with the help of Google maps reported the incidence of NDM-1 positive cases from 2009 to December 2012. It reported around 950 isolates of NDM1 producing bacteria from around the world. There were 66 articles describing imported human infection isolates with 153 (16.1\%) isolates of NDM-1 producing bacteria. Of this Klebsiella pneumoniae $(\mathrm{n}=359)$ and Escherichia coli $(\mathrm{n}=268)$ represented $37.8 \%$ and $28.2 \%$ respectively thus representing most commonly described NDM-1 producing bacteria. NDM-1 production has also been reported in Acinetobacter baumannii, Pseudomonas aeruginosa isolates and in a wide variety of non-fermenting gram-negative species [7]. Besides NDM-1 there have been reports of variants worldwide which differed at molecular level and had a different amino acid substitution due to difference in the sequence of nucleotides and had been shown to be altered further in their resistance profile. A variant of NDM-1, NDM-4 was reported in a urinary culture of a patient hospitalised in India in January 2010 in E. coli 15 isolate in which there was a single amino acid substitution

*Corresponding author: Hemant J Vira, Principal Investigator (Scientific Officer), Advanced Centre for Treatment, Research and Education in Cancer (ACTREC) Tata Memorial Centre (TMC), Kharghar, Navi Mumbai-410 210, Maharashtra, India, Tel: +912227405000; Fax: +912227405085; E-mail: virahemant@gmail.com

Received November 24, 2017; Accepted December 01, 2017; Published December 07, 2017

Citation: Vira HJ, Bhat VG (2017) Epidemiology of NDM-1 and its Variants in Multidrug Resistant Gram-Negative Bacilli Isolated from Infection in Cancer Patients. Med Chem (Los Angeles) 7: 368-370. doi: 10.4172/2161-0444.1000482

Copyright: (C) 2017 Vira HJ, et al. This is an open-access article distributed under the terms of the Creative Commons Attribution License, which permits unrestricted use, distribution, and reproduction in any medium, provided the original author and source are credited. 
(Met154Leu) and possessed increased hydrolytic activity toward carbapenems and several cephalosporins compared to that of NDM-1. This amino acid substitution was not located in the known active sites of NDM-1, indicating that remote amino acid substitutions might also play a role in the extended activity of this MBL [8]. Another variant was reported in a patient transferred from a hospital in Serbia to the Germany, without an obvious link with the Indian subcontinent and was termed NDM-2 that had a $\mathrm{C}$ to $\mathrm{G}$ substitution at position 82 resulting in an amino acid substitution of proline to alanine at position 28. The strain lacked detectable plasmids [9]. NDM-3 was identified encoded in a plasmid from Japan in a multidrug-resistant Escherichia coli isolate obtained from the faeces of a patient. The variant had an amino acid substitution at position 95 from Asp to Asn [10].

\section{National Status}

Since the first reported case of NDM-1 from India in 2009 there have been an increasing number of infections in patients from India. Kumaraswamy et al. reported that not all patients infected with NDM1 positive bacteria have a history of hospital admission in India, and extended spectrum beta lactamases are known to be circulating in the Indian community through drinking water and seepage samples [4]. A group of scientists collected around 171 seepage samples and 50 tap water samples from New Delhi and 70 sewage effluent samples from Cardiff waste water treatment works and subjected to analysis for the presence of NDM-1 gene. They found that there are two of 50 drinking water samples that contained bla (NDM-1) and 51 of 171 seepage samples from New Delhi. Thus presence of NDM-1 $\beta$-lactamase producing bacteria in environmental samples had important implications on immunocompromised cancer patients undergoing radio and chemotherapy. Deshpande et al. reported 24 consecutive carbapenem resistant Enterobacteriaceae isolates from patients admitted in a tertiary care hospital in Mumbai, India from August 2009 to November 2009 of which 22 were confirmed NDM producers as they were positive for NDM by PCR [11]. A worldwide study carried out in two hundred and thirty five isolates of Enterobacteriaceae from intraabdominal infection selected on the basis of their reduced susceptibility to ertapanam (breakpoints MICs $\geq 0.5 \mathrm{mg} / \mathrm{L}$ ) showed that a large proportion of isolates originated from India (68\%). Also of this all the thirty- three bla NDM-1-carrying isolates were from patients in India and comprised five different species [12]. A study carried out in India in 464 isolates, 57 (12.3\%) showed a reduced susceptibility to imipenem and meropenem and further showed that all the carbapenamase resistant isolates were bla (NDM-1) positive by PCR and 13 isolates bore variants that differed in sequence from bla (NDM-1) which were NDM-5, NDM-6, and NDM-7 [13]. In a study carried out in tertiary sector in south India sixty one carbapenem resistant clinical isolates of a total of $212 P$. aeruginosa isolates cultured during the study period were screened for the presence of NDM-1 by PCR. Of this NDM-1 was detected in four isolates which indicated the presence but a lower prevalence of NDM-1 in P. aeruginosa [14].

\section{Rationale}

Due to lack of epidemiological data within India, the exact prevalence of NDM-1 enzyme is not known. Also a review by Walsh et al. has highlighted the importance of NDM-1 spread and its origins in India [15]. The studies available are mostly for the detection of phenotypic characteristics for screening of carbapenem resistant isolate by antibiotic sensitivity testing. The modified Hodge test is used for detection of carbapenemase production. It is generally more sensitive for detection of carbapenemases of Ambler class A and class D [16]. It also has other limitations apart its sensitivity to detect class
B carbapenemases. These limitations include the type of carbapenem optimal for confirmation of carbapenemase production, where imipenem appears to be least specific agent [17]. In short, the modified Hodge test is an easy and expensive tool that can be used while screening for carbapenemases following to antimicrobial susceptibility tests. The limitations are that it is time consuming requiring at least $72 \mathrm{hrs}$ for result interpretation and low sensitivity and specificity for NDM-1 producers. Moreover it cannot be used as a confirmatory test for detection of NDM-1 production. All such limitations demands for the development of molecular tools for the detection and confirmation of NDM-1 producers at the genetic level. One such tool is development of Polymerase Chain Reaction (PCR) test for the specific gene encoding NDM-1 enzyme. It is the most specific method of confirmation and also most sensitive of the order of $100 \%$. Further it may be followed by sequencing to find out the variants and there effects on the NDM-1 gene. In a study by Stuart et al., this system had $100 \%$ specificity and 97\% sensitivity in detecting NDM-1 producing enterobacterial isolates. Rapid and specific detection of NDM-1 by molecular PCR technique will allow rapid antibiotic interventions for immunocompromised cancer patients and efficient use of carbapenem antibiotics [18].

\section{Future Prospects}

There is an urgent need for the development of a novel class of antibiotics and drugs against resistant gram-negative bacteria isolated in immune compromised patients such as cancer patients. Due to the increase in extended spectrum carbapenem resistance nationally as well as internationally the world is facing an increased demand for the development of various classes of antibiotics through various pharmacotherapeutical technologies. The various pharmaceutical techniques have a potential to develop a world class pharmaceutical drug capable of eradicating the threat of NDM-1 producing microorganisms. The various drug delivery platforms and drug designing methods can cater to the increase in demand of a novel drug which is difficult to develop resistance against by microorganisms.

Thus, the future of pharmacotherapeutics holds a tremendous scope offered by pharmacological drug designing platforms which has the potential to transform the precision, sensitivity and specificity of pathogen eradication in a rapid and cost-effective manner.

\section{Competing Interests}

None declared.

\section{References}

1. Walsh TR (2010) Emerging carbapenemases: A global perspective. Int J Antimicrob Agents 36: S8-S14.

2. Yong D, Toleman MA, Giske CG, Cho HS, Sundman K, et al. (2009) Characterization of a new metallo- $\beta$-lactamase gene, bla NDM-1, and a novel erythromycin esterase gene carried on a unique genetic structure in Klebsiella pneumoniae sequence type 14 from India. Antimicrob Agents Chemother 53 5046-5054.

3. Livermore DM, Mushtaq S, Warner M, Zhang J, Maharjan S, et al. (2011) Activities of NXL104 combinations with ceftazidime and aztreonam against carbapenemase-producing Enterobacteriaceae. Antimicrob Agents Chemother 55: $390-394$

4. Kumarasamy KK, Toleman MA, Walsh TR, Bagaria J, Butt F, et al. (2010) Emergence of a new antibiotic resistance mechanism in India, Pakistan, and the UK: A molecular, biological, and epidemiological study. Lancet Infect Dis 10: $597-602$.

5. Nordmann P, Poirel L, Mark AT, Timothy RW (2011) Does broadspectrum ??-lactam resistance due to NDM-1 herald the end of the antibiotic era for treatment of infections caused by Gram-negative bacteria? J Antimicrob Chemother 66: 689-692. 
Citation: Vira HJ, Bhat VG (2017) Epidemiology of NDM-1 and its Variants in Multidrug Resistant Gram-Negative Bacilli Isolated from Infection in Cancer Patients. Med Chem (Los Angeles) 7: 368-370. doi: 10.4172/2161-0444.1000482

6. Hammerum AM, Toleman MA, Hansen F, Kristensen B, Lester CH, et al. (2010) Global spread of New Delhi metallo-??-lactamase 1. Lancet Infect Dis 10: 829-830.

7. Berrazeg M, Diene SM, Medjahed L, Parola P, Drissi M, et al. (2014) New Delhi metallo-beta-lactamase around the world: An ereview using google maps. Eurosurveillance 19: 1-14.

8. Nordmann P, Boulanger AE, Poirel L (2012) NDM-4 metallo- $\beta$-lactamase with increased carbapenemase activity from Escherichia coli. Antimicrob Agents Chemother 56: 2184-2186.

9. Kaase M, Nordmann P, Wichelhaus TA, Gatermann SG, Bonnin RA, et al. (2011) NDM-2 carbapenemase in Acinetobacter baumannii from Egypt. J Antimicrob Chemother 66: 1260-1262.

10. Tada T, Miyoshi-Akiyama T, Shimada K, Kirikae T (2014) Biochemical analysis of metallo- $\beta$-lactamase NDM-3 from a multidrug-resistant escherichia coli strain isolated in Japan. Antimicrob Agents Chemother 58: 3538-3540.

11. Deshpande P, Rodrigues C, Shetty A, Kapadia F, Hedge A, et al. (2010) New Delhi Metallo-beta lactamase (NDM-1) in Enterobacteriaceae: treatment options with carbapenems compromised. J Assoc Physicians India 58: 147-149.

12. Lascols C, Hackel M, Marshall SH, Hujer AM, Bouchillon S, et al. (2011) Increasing prevalence and dissemination of NDM-1 metallo-blactamase in India: data from the SMART study (2009). J Antimicrob Chemother 66: 1992-1997.
13. Rahman M, Shuklaa SK, Prasada KN, Ovejeroc CM, Patia BK, et al (2014) Prevalence and molecular characterisation of New Delhimetallobeta-lactamases NDM-1, NDM-5, NDM-6 and NDM-7 inmultidrug-resistant Enterobacteriaceae from India. International Journal of Antimicrobial Agents 44: 30-37.

14. Shanthi M, Sekar U, Kamalanathan A, Balaraman S (2014) Detection of New Delhi metallo beta lactamase-1 (NDM-1) carbapenemase in Pseudomonas aeruginosa in a single centre in southern India. Indian J Med Res 140: 546-550.

15. Walsh TR, Toleman MA (2011) The new medical challenge: why NDM-1? Why Indian? Expert Review of Anti-infective Therapy 9: 137-141.

16. Vourli S, Giakkoupi P, Miriagou V, Tzelepi E, Vatopoulos AC, et al. (2004) Novel GES/IBC extended-spectrum ??-lactamase variants with carbapenemase activity in clinical enterobacteria. FEMS Microbiol Lett 234: 209-213.

17. Miriagou V, Cornaglia G, Edelstein M, Galani I, Giske CG, et al. (2010) Acquired carbapenemases in Gram-negative bacterial pathogens: Detection and surveillance issues. Clin Microbiol Infect 16: 112-122.

18. Stuart JC, Voets G, Scharringa J, Fluit AC, Leverstein-Van Hall MA (2012) Detection of carbapenemase-producing Enterobacteriaceae with a commercial DNA microarray. J Med Microbiol 61: 809-812. 